\title{
LE SENS DES PROPORTIONS DANS UNE SOCIÉTÉ EN MUTATION. LE RÊVE ET LA RÉALITÉ... DERRIÈRE LE DOS DE DIEU (UN ASPECT DU ROMAN DE ZSIGMOND MÓRICZ*)
}

\author{
HENRI DE MONTETY \\ Rédacteur à la revue Hungarian Studies \\ hmontety@mail.com
}

\begin{abstract}
Zsigmond Móricz situe dans une petite ville de la Haute-Hongrie sa parodie de Madame Bovary, où l'héroïne rêve d'adultère, mais ne le commet pas, et où elle perd, non la vie, mais seulement son innocence et sa jeunesse. Se prenant pour une bourgeoise, elle s'illusionne sur elle-même autant que sur les mérites de la bourgeoisie. Elle vit dans un monde où le principe qui tient les sociétés, c'est-à-dire le goût des proportions (gages de l'harmonie sociale), est détourné vers le maintient pur et simple de l'ordre au moyen de règles et des normes arbitraires. Pour caractériser l'attitude impétueuse du jeune juge qui courtise l'héroïne, Móricz propose implicitement la métaphore de la femme-piano : en observant sa manière de jouer, le juge constate que la jeune femme devrait être "accordée" et que lui seul est à même de le faire convenablement, à la différence des habitants de la petite ville qui sont selon lui beaucoup trop grossiers. L'écrivain oppose en vain la hiérarchie sociale et la hiérarchie naturelle. La seule hiérarchie valable, à ses yeux, est divine, mais personne n'y prend garde (on se débrouille... derrière le dos de Dieu). Móricz décrit comment, en l'absence de tout sens des proportions (sous l'emprise de l'inconscient), le sens du devoir lui-même (la recherche de l'harmonie) s'égare dans les illusions et provoque des catastrophes. Au point que seul le retour des normes conventionnelles et de la hiérarchie sociale arbitraire peut rétablir le calme. Exit la nature. Exit la jeunesse et la beauté.
\end{abstract}

Mots-clefs : Zsigmond Móricz, réalisme littéraire, Bovarysme, condition de la femme, inconscient

Dans une province de Haute-Hongrie, au tournant du siècle, un instituteur sans talent s'est marié avec une femme trop belle et trop jeune pour lui. Naturellement, celle-ci est courtisée de toutes parts. De toute la société masculine locale, mais aussi du jeune cousin de son mari, l'écolier dénommé Laci, et bientôt d'un jeune juge récemment nommé dans la petite ville où se déroule le drame. Un chassé-croisé doublé d'un mauvais hasard permet bientôt de croire que l'épouse

* Toutes les références entre parenthèses correspondent à l'édition française : Zsigmond Móricz, Derrière le dos de Dieu, Editions Ibolya Virág, 1996, 190 pages

Hungarian Studies 33/2(2019) 0236-6568/\$20 C Akadémiai Kiadó, Budapest 
a succombé à un étudiant en théologie de passage, venu dîner chez l'instituteur. En fait, il n'en est rien. « Brute ! s'est-elle écriée. Je ne suis pas votre petite ! Je ne suis pas une caissière ! On m'appelle madame ! » (57) L'adultère, à proprement parler, n'était pas loin d'être consommé (« la main de l'étudiant la pénétrait » écrit sobrement Zsigmond Móricz, tout en soulignant, à la décharge de la victime, la promptitude et la brutalité des préliminaires). La femme a finalement eu la force de repousser son agresseur. Tout de même, d'une certaine manière, le mal a été fait. Car elle a eu le temps d'éprouver un " plaisir aigu », une « ivresse vertigineuse » et enfin « l'amertume». (Ibid.) Tout cela en moins d'une seconde, au cours de laquelle elle a aussi eu le loisir de raisonner : elle a eu pleinement conscience que personne ne pourrait les surprendre, que l'étudiant (complètement ivre au moment des faits) aurait lui-même tout oublié le lendemain. Et pourtant, elle a renoncé, avec pour principal argument le fait qu'elle est une dame et non une caissière. Entre deux mondes, celui de la bourgeoisie et celui des petits employés, elle penche vers celui de la bourgeoisie. Et cela, au prix de son plaisir et de sa santé. C'est une illusion, bien sûr. Et même une double illusion. La première illusion n'est pas sincère, car la femme sait bien qu'en réalité, elle n'appartient pas à la bourgeoisie. La seconde illusion est véritable : elle ne sait rien du mode de vie des bourgeoises, notamment de leur vie sentimentale.

Du reste, sa réputation est provisoirement sauvée par le faux-témoignage du jeune cousin de son mari, qui prétend avoir lui-même conduit l'étudiant ivre-mort dans son lit. Il fait preuve de courage et d'abnégation (d'aveuglement, peut-être), car son mensonge compromet gravement son avenir. « Tu peux désormais considérer l'affaire jugée, dit le proviseur, tu es chassé de tous les lycées du pays. » (87)

\section{La province}

Ce genre d'histoire, selon Zsigmond Móricz, ne peut arriver qu'en province, aussi offre-t-il à son lecteur une peinture pittoresque de la vie telle qu'elle s'y passe, rétrécie à quelques rues boueuses où l'aventure se limite à une poignée de destinations possibles où l'on croise toujours les mêmes personnes. Un imbroglio de calculs, d'apparences, d'humiliations et de rapines, de complicités aimables, de goinfrerie, d'ivrognerie et d'intempérance, conjugués avec le goût plus typiquement hongrois du désordre dans l'allégresse et l'affliction.

Tout cela cohabite, bien sûr, avec le respect d'un certain nombre de normes sans lesquelles il n'y aurait pas de société. Par exemple, la hauteur du chapeau quand on salue ou la force de la poignée de main pour exprimer la cordialité ou le respect. L'instituteur, tout benêt qu'il est en moult circonstances, sait aussi comment regarder de haut un collègue, simplement parce que ce dernier a été nommé par interim. La plupart des chicanes qui ont court dans la petite ville, qui 
sont nombreuses, pourraient finalement se résumer à un problème de proportion. Autrement dit, de justice. Justement, le roman commence, en quelque sorte, avec l'entrée en scène d'un jeune juge qui vient d'être nommé. Pour lui, tout semble nouveau ici. Et son tempérament, tout comme sa formation juridique, l'incite à intellectualiser les choses. Ayant rencontré l'instituteur en compagnie de quelques autres piliers locaux d'un petit café, il décide de le nommer : Bovary. Cependant, il a bien conscience que la parodie est fort éloignée du modèle. « Pour atteindre un tel développement de soi-même, il faut une société très civilisée. Quelle caricature de caricature où nous vivons... (...) Là-bas, la vie est odorante, colorée... Mais ici tout est si froid et terne. » (18) Notons que Móricz veut peut-être attirer notre attention sur une certaine déficience de jugement chez le jeune juge, auteur de ce raisonnement. De toute évidence, ce dernier s'illusionne sur la nature odorante et colorée de la province française. (Sinon, pourquoi Flaubert aurait-il écrit madame Bovary ?) Quant à la maison de l'instituteur, il serait possible, pour la décrire, d'employer par exemple l'expression de kermesse bariolée.

Du reste, la maison de l'instituteur n'est pas la Hongrie, ni même la petite ville de Haute-Hongrie. Une petite ville est à elle-seule tout un monde. Le roman de Móricz en décrit la structure et surtout les barrières sociales, infranchissables, qui constituent aussi les conditions même de l'intrigue.

\section{La limite entre l'illusion et la vérité}

Dès la première page du livre, l'instituteur exprime son insatisfaction au sujet de sa situation sociale. Tandis que trois longues charrettes l'empêchent de traverser une rue, il grommelle : "Même les voitures se mettent en travers de mon chemin !» (7) Ce chemin, selon lui, c'est sans doute celui qui le ferait grimper jusqu'à la position de professeur ou même, qui sait, d'inspecteur. Il est, à proprement parler, hors de lui : les règles et normes qui pèsent sur lui, à n'en pas douter, lui ont fait perdre le sens des proportions. Dans le même temps, la cible de son mécontentement se déplace du ministère à d'innocentes charrettes. Le transfert permet à l'instituteur de penser à ses problèmes de manière moins réaliste. Ce qui est beaucoup plus reposant. Móricz Zsigmond souligne que son personnage tient « par dessus tout à éviter les idées noires. » (Ibid.)

Ce mode de pensée, d'ailleurs, est au fondement des règles et des normes qui gouvernent la petite ville. On évite les problèmes. Et tout est question de point de vue. Au point que la loi, en définitive, en devient absolument arbitraire. Ainsi du contraste entre la sévérité du proviseur à l'égard des lycéens et son indifférence en ce qui concerne le comportement de l'instituteur et du licencié en théologie ; contraste qu'il serait peu subtil de considérer comme de la simple hypocrisie. Il s'agit plutôt d'un mode de pensée cohérent, fondé sur le cloison- 
nement. L'instituteur s'en fait l'écho, à son niveau, avec une candeur touchante. Au cours d'une discussion avec un collègue, il s'emballe et tient un discours aux limites de la schizophrénie : "Dans la vie, on n'a précisément besoin que des choses que l'on n'a pas apprises à l'école. Le plus important, c'est l'amour! Puis gagner de l'argent! Eh bien, cela serait du propre, si l'on voulait l'apprendre à l'école ! » (29) Notons que si la question de l'amour demeure pour lui théorique, celle de l'argent a été résolue - à son très modeste niveau - grâce à une coopérative d'achat qu'il a fondée et dirige largement à son propre profit.

L'argent aurait pu être le sujet du livre : ce sera l'amour. Par conséquent, l'instituteur n'y est pas à son avantage. L'instituteur est écrasé par son épouse. Elle sait vivre, elle est plus intelligente que lui et a toujours un temps d'avance. Parfois, lui-même s'en rend compte. Ainsi lui arrive-t-il de sentir « qu'elle avait encore raison. » (22) Le juge ne tarde pas à remarquer le caractère exceptionnel de la femme. Précisément, il change d'avis sur elle quand il apprend qu'elle a loué Dieu le jour où ses pourceaux sont morts. «C'est une dame!" se dit-il. Être une dame, là est la question. " D'un coup de baguette magique, la vie banale était écartée. » (21) Du reste, n'est-ce pas encore une illusion? Le jeune juge devrait le savoir, lui qui est en mesure de fréquenter de vraies dames, des filles et des femmes d'avocat, de procureur. Ce jeune homme, à vrai dire, est à la fois proche et loin de la vérité. Il a, d'ailleurs, juste ce qu'il faut de lucidité pour se rendre compte de son état intermédiaire et de l'état intermédiaire de tout ce qui l'entoure, c'est-à-dire à la limite du mensonge et de la vérité, du prototype et de la caricature, de l'ordinaire et de l'extraordinaire. En voyant le boucher qui a «l'allure d'un boucher de journal humoristique », il formule intérieurement la pensée suivante. « Il y a beaucoup de choses curieuses dans cette petite ville, pourtant, il n'y manque pas de choses banales. » (95) Finalement, n'est-ce pas le cas de toutes les petites villes (en Hongrie comme en France) ? Tout est question de point de vue. Pour le juge, c'est la rencontre de la femme de l'instituteur qui va modeler, au moins provisoirement, son point de vue sur les choses.

Quant à une sommité de la ville, comme peut l'être le proviseur, son point de vue ne devrait pas être aussi mobile, car il est solidement fixé aux règles et aux normes qui gouvernent la petite ville. Et pourtant, on s'aperçoit peu à peu que le proviseur adopte une position plus souple à l'égard du jeune Laci qu'il menaçait d'abord d'expulsion. Il apparait finalement qu'une « petite retenue » ferait l'affaire (87). C'est qu'entre temps, le bruit a couru que la femme, risquant d'être éclaboussée dans le scandale, menace de quitter la petite ville. il ne faudrait pas que les règles et normes se retournent contre la loi (de l'arbitraire), qui est avant tout le bon plaisir des notables. 


\section{La femme-piano : point d'orgue du roman}

À la loi d'airain fixée par les relations sociales, Zsigmond Móricz oppose les lois de la nature. Ou peut-être celles de l'art ou celles de l'amour. On n'est pas obligé, pour l'heure, de trancher sur de subtiles distinctions. Il suffit de comprendre qu'il s'agit essentiellement d'une réaction contre l'oppression. Et d'une métaphore en forme de chimère : la femme-piano.

À peu près au milieu du livre, le jeune juge est invité à déjeuner chez l'instituteur. En attendant que le repas soit servi, la femme s'installe au piano. L'instituteur, qui va bientôt disparaître à la cave pour en revenir un peu plus tard avec trois bouteilles dans chaque main, s'intéresse peu à la musique. Et si le piano sonne un peu faux, cela lui est bien égal. Il décrit, à cet égard, le raisonnement des parents dont les enfants viennent ici prendre des leçons. « Les parents ne laissent pas toucher au piano, chez eux (...). Ils sortent toujours cet argument : pour apprendre, un mauvais piano suffit, et il serait dommage de détraquer le bon piano que l'on a chez soi, alors que, celui de Monsieur l'instituteur, cela n'a pas d'importance... » (106) Après avoir achevé cette brillante analyse, il désigne le vieux piano en disant que pour celui-ci, effectivement, " cela n'a pas d'importance. » (Ibid.) Autrement dit, il reproche aux parents (bourgeois) qui le dominent un raisonnement qu'il fait lui-même : définition même de l'aliénation. Pour exprimer cela dans la terminologie de la présente étude, je dirais que nous avons affaire à une illusion que l'instituteur veut s'imposer à lui-même au sujet des proportions. (L'accordage d'un piano tout comme l'harmonie du monde étant des signes de la bonne proportion des mesures.)

Voici comment Móricz décrit le concert improvisé. « La femme ouvrit le piano, s'assit devant le clavier et, sans partition, attaqua un morceau. (...) Les doigts raides, allongés, comme avec des baguettes, (elle) frappa les touches qui rendirent une musique désespérée et un peu confuse. Cette façon de jouer était, en quelque sorte, miraculeuse, aussi rapide qu'une charge de hussards. Une cacophonie d'un caractère merveilleux éclata, sans un son juste, mais qui sembla au juge, plus admirable qu'aucune musique entendue jusqu'alors. » (Ibid.) Après un premier morceau de musique classique, la femme joue des airs populaires. Ce qui permet à Móricz de reprendre le même type de description cocasse. Le juge, quant à lui, trouve la pianiste de plus en plus jolie. « Ses doigts fins, et droits comme des flèches, frappaient si étourdiment et avec tant d'insouciance les touches jaunies par le temps, que cela semblait la chose la plus naturelle du monde. » (107) Nous savons que le juge pratique volontiers les associations d'idée douteuses, à la fois vraies et fausses ou plutôt à la limite entre les deux. En l'occurence, ce spectacle lui fait penser que « quelque chose s'est passé dans la nuit». (Ibid.) Sans pitié, il cherche du coin de l'oeil « des ornements sur le front de l'instituteur ». (Ibid.) Or il s'agit bien du lendemain de la nuit où l'étudiant en théologie, ivre mort, est 
resté dormir dans la maison de l'instituteur. Mais le juge commet une erreur, car ce qui s'est passé n'est pas ce qu'il croit. D'ailleurs, son intuition lui dit aussi que les choses, en général, ne sont pas aussi simples qu'elles en ont l'air. Il remarque, par exemple, que les « jolies petites mains blanches » de la femme donnent l'impression d'une « exhibition de force». (108) Ce paradoxe nous rappelle une nouvelle fois que le juge a coutume des phénomènes incertains ou intermédiaires. Justement, Móricz nous apprend que ce petit concert est l'occasion, pour lui, de se fondre dans l'atmosphère de la pièce. Bientôt c'est dans l'âme de la petite ville qu'il pénètre. Comme dans une énigme, l'énigme de la femme-piano...

\begin{abstract}
«Il ne parvenait pas à comprendre l'uniformité de son jeu. Toutes ses chansons semblaient avoir le même accompagnement. (...) Soudain il découvrit le secret. La femme avait admirablement résolu le problème de la musique pour toute sa vie. Elle avait inventé une véritable "clef de magicien" qui lui permettait de régaler, sans limite, ses invités de musique populaire. Avec la main droite, elle jouait en solo le "chant", et, avec la main gauche, elle plaquait l'accord parfait correspondant à chaque note, autant, du moins, qu'elle-même et le piano en étaient capables. (...) Le juge sourit largement et donna raison à la femme. Ici, toute la vie était simplifiée. Tout raffinement, tout artifice était banni. Il n'y avait qu'à découvrir la clef, et tout devenait limpide comme un ruisseau. On voit chaque pierre au fond du ruisseau; et qui donc oserait dire que le ruisseau n'est pas délicieux ? (...) Il ferma les yeux et songea aux trésors qui s'égarent en des mains indignes. (...) Jamais il ne vient un artiste pour jouer sur l'âme de ces femmes la symphonie de l'amour. (...) Et elle ne se doute pas qu'elle est, elle, toute pareille à ce vieux piano abîmé par tant d'élèves aux mains novices, - et à qui la visite d'un accordeur ne serait pas inopportune. Avec mélancolie et un peu de souffrance, il sentait combien volontiers il se fût chargé de ce rôle d'accordeur. » (Ibid.)
\end{abstract}

En supposant que la femme-piano soit à son tour une femme-monde, il y a deux idées majeures dans ce passage central du livre. D'une part, le fait que l'harmonie fragile et naïve du monde (l'authentique absence de proportions) est la plupart du temps réduite en bouillie par la brutalité d'individus sans délicatesse qui croient pouvoir imposer leur propre mesure à toutes choses, imposer une hiérarchie, sociale ou technique, c'est-à-dire des proportions impropres à l'harmonie. D'autre part, en s'imaginant lui-même dans la position d'un accordeur de piano, le juge se croit capable de rétablir l'authentique harmonie dans le monde et s'en donne même la mission. Il croit, en quelque sorte, pouvoir et devoir substituer des proportions artificielles à l'absence authentique de proportions. Pour cela, il devrait cesser incontinent de penser en terme de hiérarchie. Mais c'est impossible. En l'occurence, il met en première place son propre intérêt : son objectif est d'obtenir la femme de l'instituteur. En se parant des plumes de la justice (rendre à cette 
femme la place qu'elle mérite), il s'enfonce dans l'illusion. Finalement, il ne dépasse pas le niveau des habitants de la petite ville.

\section{Le sens des proportions}

Le jeune juge ne manque pas de vanité. Non moins de naïveté. D’ailleurs, ébranlé par le concert de piano, il continue à se sentir dans le même état intermédiaire qui semble propre à l'inspirer. Il s'agenouille bientôt devant la femme de l'instituteur, empli de confusion, convaincu qu'il est aux pieds de sa maîtresse, de son épouse ou alors de sa mère. "Une volupté, un rêve chaleureux s'emparait de lui, sans qu'il s'en rendît bien compte. Il se dit alors : "Le romantisme ne meurt jamais en nous." » (109-110) Elle joue pour lui une vieille chanson. " Des larmes jaillirent des yeux du juge. Il ouvrit les bras et enlaça le beau cou de la femme. » Est-ce le début d'une romance ? Non. Zsigmond Móricz renoue avec la scène de séduction ratée (comme avec le licencié de théologie), mais cette fois-ci sur un mode plus conscient, moins violent - et donc plus ironique. À la différence de l'étudiant, le juge se comporte avec décence et répond au regard ironique de la femme s'écartant de lui par le même regard : ironie sur lui-même. Du reste, il peut se le permettre, car il est persuadé d'arriver à ses fins plus tard. « Naturellement, se dit-il, cette musique a une clef très simple. Il faut toujours commencer par le commencement, et continuer ensuite... Ne jamais commencer par la fin!» (111) Commencer par le début, c'est sans doute ce que la femme considère comme un comportement bourgeois, comme ce qui convient à une « dame». Patience...

Pendant ce temps (ou plutôt depuis le début du livre), l'instituteur « se tire le nez qu'il a long ». Encore un problème de proportions qui tardent à s'établir. Un problème essentiel, selon Móricz, qui prend la peine de souligner que ce nez est celui des « anciens Hongrois ». (7)

Vers la fin du roman, après une scène de dispute collective, le juge et la femme se retrouvent seuls dans une pièce. Ils s'enlacent. Mais Laci entre dans la pièce à l'improviste. Il disparaît aussitôt. Suivi par le juge, qui décide sans regrets d'abandonner la place. Il est envahi de dégoût, car il a tout d'un coup le sentiment de s'être mêlé à l'ordure (avec ce terme, il désigne ses nombreux concurrents - pas seulement Laci, mais, en l'occurence, ce dernier a fait une apparition de trop au mauvais moment). Le juge ne regrette pas son échec. Pour se consoler, sommet de la rationalisation, il se convainc que l'opération aurait été trop facile. Il va plutôt se tourner vers les familles notables de la petite ville. En un court raisonnement, il remet tout le monde à sa place et rétablit la hiérarchie artificielle (sociale), que l'on a cru un instant supplantée par quelque chose qui ressemblait à une hiérarchie naturelle, relevant de la séduction et du désir animal. 
Pourquoi Zsigmond Móricz a-t-il dissimulé cet instant « derrière le dos de Dieu »? Sans doute en raison du fait que la seule hiérarchie valable n'est ni sociale, ni naturelle. Elle est divine. La hiérarchie divine est seule à pouvoir procurer une harmonie dépassant toute notion de proportions, donc de calcul, d'intérêt, de désir et de plaisirs, d'envie de domination d'aucune sorte. Toute ce qui arrive dans la petite ville se passe « derrière le dos de Dieu ».

Il est vrai que la hiérarchie naturelle, au moins, entretient une relation avec la réalité, aussi imparfaite soit-elle. La hiérarchie sociale, quant à elle, est tout-à-fait artificielle. Et le juge a besoin de toute sa force de conviction pour se convaincre lui-même qu'il ne s'est que trop mêlé à l'ordure. Il se retire de la nature avec dignité (selon lui), pour aller se réfugier dans les zones (selon lui) plus aérées de la société. Mais n'a-t-il pas, lui-même, été ordurier ? Croyant que ses calculs sordides lui procuraient une lucidité absolue, il était plongé dans le royaume de l'inconscience. Quelque part dans les zones intermédiaires que Zsigmond Móricz se plait à explorer, là où l'illusion se manifeste sous la forme d'une réalité impérieuse. La plus simple de ces zones intermédiaires, c'est la boisson et l'ivresse. Plus pervers et tout aussi trompeur : l'étrange domaine de l'inconscient. Enfin, à rebours, mais non moins source d'illusions : le sens du devoir.

\section{L'illusion et la réalité - la boisson, l'inconscient et le sens du devoir}

En toute circonstances, l'homme a la nostalgie de Dieu. Il est prêt à tout pour se cacher qu'il est une vile créature vivant de calculs et d'expédients. Si le monde ancien procurait, naguère, de large espaces d'harmonie où les proportions n'avaient pas cours, le monde moderne les a, pour ainsi dire, réduite à leur plus simple expression. Ainsi le goût pour le Moyen âge est-il une sorte de nostalgie de la nostalgie.

Zsigmond Móricz écrivait au temps où la modernité déferlait sur l'Europe centrale. Avec les premiers vendeurs d'assurance et de parapluies, le calcul faisait son entrée dans les campagnes, non pas comme ce qu'il avait toujours été, un mal nécessaire qui pèse lourd sur l'innocence, mais comme un mode de vie revendiqué, un nouveau modèle qu'il faut suivre si l'on veut faire comme tout le monde. Et désormais, il était impératif de faire comme tout le monde.

Móricz explore l'idée de la limite entre l'illusion et la réalité, l'idée d'absence ou au moins de déformation des proportions, c'est-à-dire du sens de la hiérarchie. Et cela pas seulement à propos du juge.

L'instituteur, depuis longtemps (on ne sait pas depuis quand), s'est réfugié dans le monde des illusions. C'est à peine si le vin lui est nécessaire. De temps en temps, la réalité vient lui rappeler quelque vérité désagréable, mais nous savons déjà qu'il fait tout pour éviter les « idées noires ». La boisson, tout de même, y a 
une part. Móricz ne ménage pas ses efforts pour nous faire comprendre quel genre d'ivrogne il est. Avalant verre après verre dans l'estaminet de la ville, remontant de la cave chargé de bouteilles, endormi sur sa chaise avant la fin du dîner... Au plus fort du drame, face à une assemblée d'homme tétanisés par l'effroi ou la honte, il a encore la force ou le toupet de s'écrier : « Haut les verres ! Nous allons nous amuser, maintenant, comme Dieu en France ! (156) D’ailleurs, la passion de l'ivresse coexiste chez lui avec le goût de la bonne chère. Un autre jour, d'un morceau joué au piano par son épouse, il admet, tout joyeux, qu'il évoque pour lui le «gazouilli des fourchettes. » (107) Peut-être, au fond, qu'il aimerait être plus à l'unisson de sa femme ? Móricz nous signale qu'il invite souvent celle-ci à boire avec lui. Sous-entendu : il n'est pas en mesure de lui offrir un autre mode de communion conjugale. N'empêche, il montre en cela un tempérament généreux qu'il manifeste, par ailleurs, largement dès qu'il est question de boire et manger. $\mathrm{Au}$ fond de lui, peut-être est-il content de son sort et croit-il devoir faire partager son contentement?

Ce n'est pas le cas de son épouse. D'ailleurs, celle-ci n'est pas seulement épouse mais aussi fille d'ivrogne. C'est-à-dire qu'elle n'est pas novice : elle sait que « quand ils sont ivres, ils sont inoffensifs ». (54)

Des forces provenant du passé agissent sur le présent. Elles se manifestent dans le monde de la conscience, mais aussi dans celui de l'inconscient. Or, autant la femme est sujette aux forces obscures agissant sur le corps (migraines, fièvres, douleurs, alitements prolongés), autant l'instituteur semble, quant à lui, être le jouet de celles qui agissent sur l'esprit. Sur ce point, le juge a vu juste : l'instituteur est Monsieur Bovary. C'est lui-même qui a tenu à présenter son épouse au licencié en théologie et au jeune juge. Toujours innocemment, il incite même les deux messieurs à lui faire la cour (tandis que l'étudiant a déjà les yeux posés sur la poitrine de la femme - regard que l'intéressée, elle, a bien remarqué.) « La sagesse de l'instituteur l'empêchait de rien voir » (28) souligne Zsigmond Móricz avec perspicacité. Car c'est aussi la sagesse de l'instituteur - à comparer à la naïveté du jeune juge - qui lui fait comprendre que le roman de madame Bovary peut bien avoir lieu dans sa petit ville, même s'il ignore tout de ce roman y compris son titre. Visiblement, la lecture est sans effet sur le juge, tandis que l'instituteur bénéficie d'une sagesse ancestrale. Le seul problème, c'est que son inconscient lui joue des tours et renverse les bienfaits de cette sagesse. Il lui dicte précisément ce qu'il ne faut pas faire.

Le sens du devoir relève bien évidemment d'une autre espèce ; il peut, au contraire, être la manifestation d'une conscience aiguë. Est-il un aspect de la mesure ou de la démesure ? Derrière la prudence de la femme se trouve un certain calcul. Par contre, en ce qui concerne le jeune Laci, qui prend le risque d'être renvoyé de son lycée pour avoir (inutilement) tenté de sauver la réputation de sa tante, qu'il admire et adore d'un amour caché, il s'agit bien d'un total abandon, 
autrement dit de démesure. Mais par rapport à quel standard ? Le sens du devoir n'est-il pas, à proprement parler, un devoir, c'est-à-dire qu'il ne peut être sujet à la discussion, qu'il n'est pas comparable à une ou plusieurs solutions alternatives. Le sens du devoir n'est pas seulement démesuré, au sens commun, il est hors de proportion, il est véritablement absolu. D'ailleurs, l'attitude adoptée par Laci lui permet d'entrevoir brièvement un autre monde. L'écolier songe « qu'il serait merveilleux de répondre pour les paroles de la femme, dût le monde s'écrouler sur lui. » (88) Durant l'interrogatoire, auquel assiste son oncle, l'instituteur, ce dernier prend conscience que son neveu est un étranger pour lui : manière de dire que le jeune Laci est véritablement ailleurs. Là où le sacrifice ne se mesure pas, ne se calcule pas. Là où la justice ne se rend pas, car elle est déjà rendue. Pascal a exprimé cela : "Tu ne me chercherais pas si tu ne m'avais déjà trouvé. »

Au demeurant, l'illusion de justice (et du devoir), dans le roman de Móricz, est à l'image de l'illusion de réalité. Ainsi de la parodie jouée par les hommes mûrs de la petite ville, angoissés par la perspective de perdre l'épouse de l'instituteur si elle met ses menaces à exécution. Soudain, ils rivalisent d'imagination pour sauver le garçon (intervention au conseil de discipline, au conseil de la Caisse d'épargne, directement auprès du proviseur...). Aux mensonges, ils ajoutent de nouveaux mensonges, mais jamais ils ne s'intéressent à la vérité, à ce qui s'est véritablement passé. "Comme vous savez bien vous y prendre à faire des histoires avec rien, dit la femme. Voilà comment agissent les hommes ! Au lieu de trouver la vérité. » (125) Móricz, sarcastique, offre un instant de gloire bien méritée à la femme de l'instituteur, qui semble jouir pleinement de sa puissance. «Cet après-midi pourrait être la récompense de toute sa vie. » (140) D'ailleurs, elle n'est pas dupe et, à son grand désespoir, elle sait qu'entre elle et les autres demeure une barrière infranchissable.

\section{Dénouement - Le retour des classes sociales}

Le dénouement proposé par Zsigmond Móricz a pour vocation de refermer la parenthèse constituée par cet instant passé derrière le dos de Dieu. La hiérarchie sociale reprend ses droit sur la nature. Ce dont on pourrait, d'une certaine manière, se réjouir. Si ce n'était que la femme est la principale et même la seule victime de cette normalisation. Toujours aussi délaissée par son vieux mari impuissant, abandonnée par le juge et méprisée par Laci, auquel elle a juste eu le temps de plaquer un « baiser furieux » avant d'aller se coucher (mais celui-ci choisit d'aller assouvir sa curiosité à la maison close : pour lui, « l'idée de femme est à jamais abolie » (178)) ; face à toutes ces déconvenues, la femme de l'instituteur, toute la nuit, se tord de douleur. Móricz décrit sans vergogne ses souffrances. "Elle empoigne son jeune corps. » Peut-être est-elle atteinte d'une colique - ou de 
« quelque diablerie»? (162) Au matin, elle a ses périodes. La vie continue, ou plutôt elle reprend, avec ses peines. Entre temps, le jeune juge a paraît-il séduit la femme du notaire. Cela se sait bientôt dans toute la ville. On en parle. On ne sait pas bien ce qui s'est passé, si c'est vraiment lui ou un autre. Laci, de son côté, obtient le pardon lors d'une séance de confession collective imposée par le proviseur. Dans la maison de l'instituteur, la femme fait une scène : elle regrette de s'être mariée avec un " misérable » qui la " rend malheureuse ». Dans son élan, elle affirme qu'elle pourrait le tromper mille fois, " même le bon dieu le ne lui reprocherait pas. » (161) L'instituteur, impassible, comme si rien ne s'était passé, répond avec son bon sens que sa femme a tout le loisir de s'amuser comme aucune autre.

« Mais pas au bal de l'élite.

- Évidemment, je ne fais pas partie de cette société. » (Ibid.)

Ce court dialogue montre, si c'était besoin, que le bon sens ne permet pas de résoudre tous les problèmes. Car la situation du ménage de l'instituteur demeure modeste et n'a aucune raison de s'améliorer. En une nuit, la femme a perdu sa beauté. Quant à l'instituteur, il gémit machinalement que son ménage était mieux tenu quand il était célibataire.

Peu après, à la mode hongroise, le comique succède au tragique dans une combinaison inextricable. Tandis qu'il est au café avec ses amis, l'instituteur apprend que sa femme a sauté par la fenêtre. Mais pas de très haut, dit-on. Elle n'a presque rien, paraît-il. Le docteur souligne : " Encore heureux qu'elle est tombée sur le derrière. » (184) Rires...

\section{Derrière le dos de Dieu...}

Le jeune juge, moitié curieux, moitié lucide, se rend compte que la hiérarchie sociale, dans la petite ville, est arbitraire. La loi y est arbitraire, car les règles et les normes, négligeant toute considération sur la nature, sont mises au seul service des intérêts de l'élite sociale et mondaine. Or, sans même s'en rendre compte, le juge adopte l'attitude de ceux qu'il entend critiquer. Car il agit, tout autant qu'eux, derrière le dos de Dieu. Négligeant la gloire de Dieu (le bien commun), il pousse même la logique de l'élite jusqu'au bout, car il agit, finalement, sinon dans l'intérêt d'une caste (la bourgeoisie), pour son unique et seul intérêt. En la femme dont il est le seul à comprendre les souffrances, il ne voit qu'une proie, une femme-piano qu'il se propose d'accorder. Il est donc d'autant plus coupable. La femme est épuisée par la nature et lui, il se joue de la nature. Du reste, les circonstances lui offraient peu d'alternative. C'est l'illusion dont il se berce qui le rend coupable. Il prétend rétablir l'harmonie, quand il ne fait que substituer une forme d'illusion à une autre, un calcul sur la nature à un calcul 
d'ordre social. Dans un cas comme dans l'autre, la mesure est à l'origine de l'action. Dans le monde moderne, le calcul et la mesure sont toujours à l'origine de l'action. Zsigmond Móricz propose trois cas de figure où le calcul semble absent ou du moins secondaire, pour bien montrer dans quelle impasse cela peut conduire. (1) Illustrée par l'instituteur pour (se) cacher son impuissance, l'ivresse n'est pas une bonne solution. (2) L'inconscience (l'inconséquence), encore illustrée par l'instituteur, qui, de peur de perdre sa femme, l'offre à ses amis, n'est pas non plus une solution. (3) Reste le sens du devoir. Laci, neveu de l'instituteur, s'offre pour sauver la réputation de la femme. Il se sacrifie au péril de son avenir et sans contrepartie. Sans calcul, sans mesure. D'ailleurs, quand la contrepartie se profile inopinément (quand la jeune femme lui fait, en quelque sorte, la promesse de s'offrir à lui), le jeune Laci entre en panique et prend la fuite. Finalement, la jeune femme conserve son innocence. Dans la ville, un autre crime est commis. La justice est rendue de manière grotesque et débonnaire, à coups de mensonges. L'ordre règne. Le prix à payer, c'est que la jeune femme, sinon son innocence, a perdu la beauté qui faisait d'elle le ravissement de la petite ville. Finalement, c'est au moins cela que les bourgeois auront perdu. Mais s'en rendent-ils compte? 